\title{
Prospective Evaluation of Glenoid Bone Loss After First-Time and Recurrent Anterior Glenohumeral Instability Events
}

\author{
Jonathan F. Dickens, MD¹, Sean E. Slaven, MD¹, Kenneth L. Cameron, PhD, MPH, ATC ${ }^{2}$, Adam M. Pickett, MD², Matthew
} A. Posner, MD $^{2}$, Scot Campbell, MD³ , Brett D. Owens, MD

${ }^{1}$ Walter Reed National Military Medical Center, Bethesda, MD, USA, ${ }^{2}$ Keller Army Hospital, West Point, NY, USA, ${ }^{3}$ Brooke Army Medical Center, San Antonio, TX, USA, ${ }^{4}$ Brown University Alpert Medical School, Providence, RI, USA.

Objectives: Determining the amount of glenoid bone loss in patients following anterior glenohumeral instability events is critical to guiding appropriate treatment. One of the challenges in managing shoulder instability in young athletes is the absence of clear data showing the impact of each event. The purpose of this study was to prospectively determine the amount of bone loss associated with a single instability event, in the setting of both firsttime and recurrent instability.

Methods: We conducted a prospective cohort study of 714 athletes followed for four years. Baseline assessment included a subjective history of shoulder instability. Bilateral shoulder MRIs were obtained in all participants with and without a history of previous shoulder instability. The cohort was prospectively followed during the study period and those who sustained an anterior glenohumeral instability event were identified. A post-injury MRI was obtained and compared to the screening MRI. Glenoid width was measured for each patient's pre- and post-injury MRI. The projected total glenoid bone loss was calculated and compared for patients with a prior history of shoulder instability.

Results: Of the 714 athletes that were prospectively followed during the four-year period, 23 shoulders in 22 subjects sustained a first-time anterior instability event (5 dislocations, 18 subluxations), and six subjects with a previous history of instability sustained a recurrent anterior instability event (1 dislocation, 5 subluxations). On average, there was statistically significant glenoid bone loss $(1.84 \pm 1.47 \mathrm{~mm})$ following a single instability event $(p<0.001)$, equivalent to $6.8 \%(95 \% \mathrm{Cl}: 4.46 \%, 9.04 \%$, range $0.71 \%-17.6 \%)$ of the glenoid width. Twelve shoulders $(52 \%)$ demonstrated glenoid bone loss $\geq 5 \%, 4$ shoulders demonstrated glenoid bone loss $\geq 13.5 \%$ and no shoulders had $\geq 20 \%$ glenoid bone loss after a first-time instability event. Pre-existing glenoid bone loss in subjects with a history of instability was $10.2 \%(95 \% \mathrm{Cl}: 1.96 \%, 18.35 \%$, range $0.6 \%-21.0 \%)$. This bone loss increased to $22.8 \%(95 \% \mathrm{Cl}: 20.53 \%, 25.15 \%$, range $21.2 \%$ to $26.0 \%)$ following an additional instability event $(\mathrm{P}=0.0117)$. All six shoulders with recurrent instability had $>20 \%$ glenoid bone loss.

Conclusion: Glenoid bone loss of $6.8 \%$ was observed after a first-time anterior instability event. In the setting of recurrent instability, the total calculated glenoid bone loss was $22.8 \%$ with a high prevalence of bony Bankart lesions (5/6). The findings of this study support early stabilization of young, active subjects following a first-time anterior glenohumeral instability event.

This open-access article is published and distributed under the Creative Commons Attribution - NonCommercial - No Derivatives License (http://creativecommons.org/licenses/by-nc-nd/4.0/), which permits the noncommercial use, distribution, and reproduction of the article in any medium, provided the original author and source are credited. You may not alter, transform, or build upon this article without the permission of the Author(s). For article reuse guidelines, please visit SAGE's website at http://www.sagepub.com/journals-permissions. 

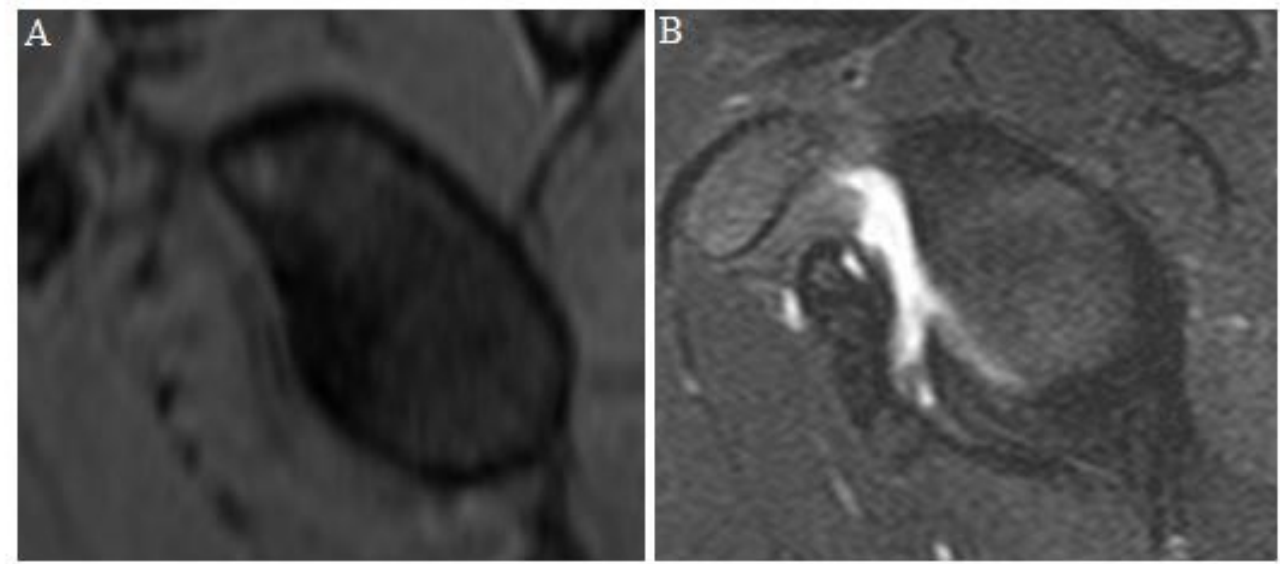

Figure 2. Clinical demonstration of a sagital en face MRI obtained prior to any instability event (A) and following a single subluxation event (B) with $14 \%$ bone loss and a bony Bankart.

Table 2: Glenoid Bone Loss in First-time and Recurrent Instability Cohorts

\begin{tabular}{|l|l|l|l|l|l|}
\hline $\begin{array}{l}\text { Instability } \\
\text { Type }\end{array}$ & $\begin{array}{l}\text { No. } \\
\text { Shoulders }\end{array}$ & $\begin{array}{l}\Delta \text { Glenoid Width } \\
(\mathrm{mm})\end{array}$ & $\begin{array}{l}\Delta \text { Glenoid Width } \\
(\%)\end{array}$ & $\begin{array}{l}\text { Total Glenoid Bone } \\
\text { Loss* }\end{array}$ & $\begin{array}{l}\text { P } \\
\text { value }\end{array}$ \\
\hline First-time & 23 & $1.85 \pm 1.49$ & $6.8 \%$ & $6.8 \%$ & $<$ \\
\hline Recurrent & 6 & $3.75 \pm 2.37$ & $13.6 \%$ & $22.8 \%$ & .0001 \\
\hline
\end{tabular}

The Orthopaedic Journal of Sports Medicine, 6(7)(suppl 4) DOI: $10.1177 / 2325967118 S 00096$

(CThe Author(s) 2018 